Supporting Information

\title{
Post-treatment of Perovskite Films towards Efficient Solar Cells via Mixed Solvent Annealing
}

Liuwen Tian, Wenfeng Zhang, * Hua Yu, * Changtao Peng, Hanying Mao, Yuepeng Li, Qiyun Wang, Yuelong Huang*

Institute of Photovoltaic, Southwest Petroleum University, Chengdu, 610500, P. R. China

\section{Corresponding Author}

*E-mail: wfzhang@mail.ustc.edu.cn (W.F.Z.).

*E-mail: h.yu2@uq.edu.au (H.Y.).

*E-mail: huangyl@vip.163.com (Y.L.H.). 


\section{Supporting figures and tables:}

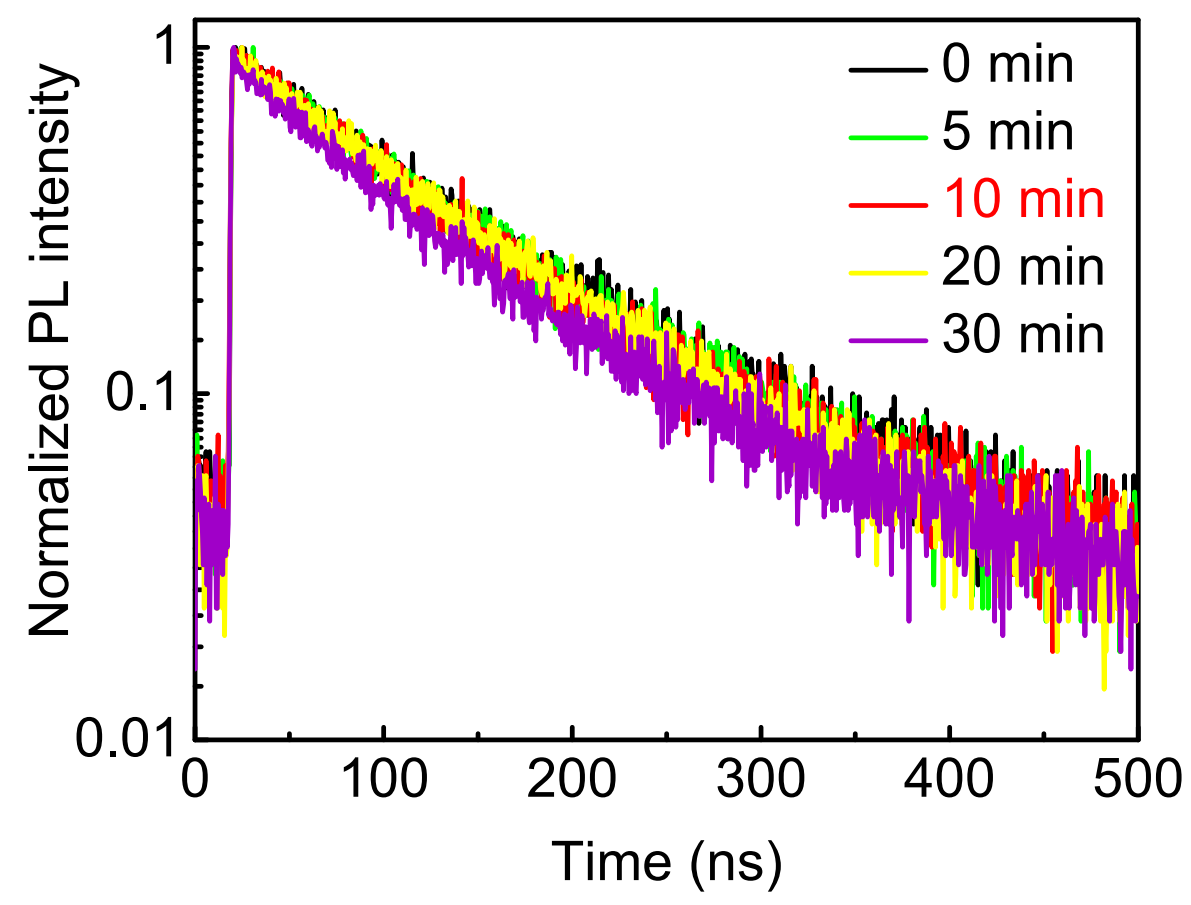

Figure S1. Time-resolved photoluminescence spectra (TRPL) of perovskite films with different thermal annealing time at $100{ }^{\circ} \mathrm{C}$ after solvent annealing at $80{ }^{\circ} \mathrm{C}$ for $10 \mathrm{~min}$.

Table S1. Summary of parameter data from TRPL results of Figure S1.

\begin{tabular}{cccc}
\hline Annealing time & $\tau_{1}(\mathrm{~ns})$ & $\tau_{2}(\mathrm{~ns})$ & $\chi^{2}$ \\
\hline $0 \mathrm{~min}$ & 41.3 & 119.5 & 1.011 \\
$5 \mathrm{~min}$ & 86.5 & 144.7 & 1.001 \\
$10 \mathrm{~min}$ & 92.2 & 343.3 & 1.035 \\
$20 \mathrm{~min}$ & 58.1 & 118.6 & 0.941 \\
$30 \mathrm{~min}$ & 53.5 & 119.0 & 1.002 \\
\hline
\end{tabular}




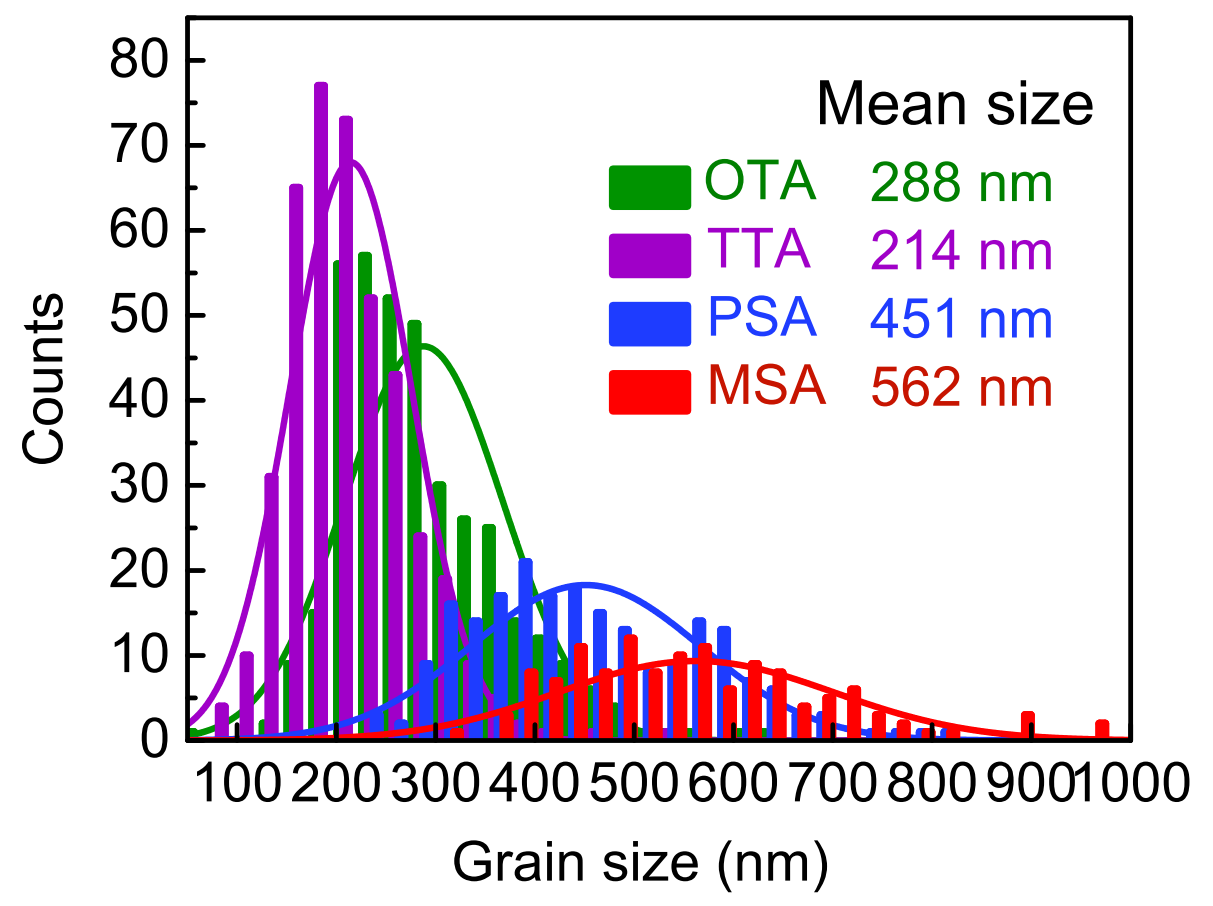

Figure S2. Grain size distribution of perovskite films with thickness of $\approx 500 \mathrm{~nm}$ (calculated from Figure 2a-d).

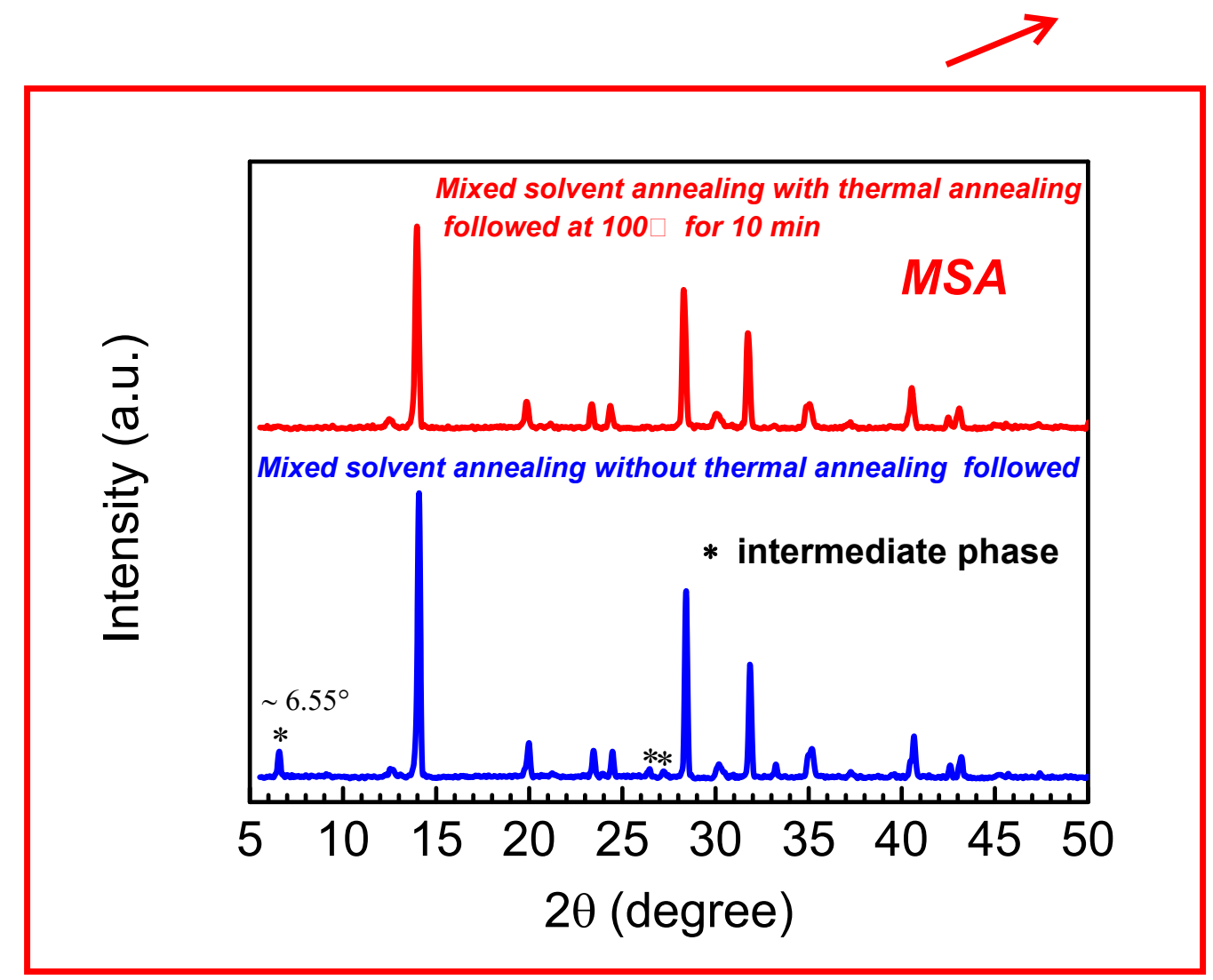

Figure S3. XRD patterns of mixed solvent annealing treated $\mathrm{MAPbI}_{3}$ films with 
(red) or without (blue) thermal annealing followed
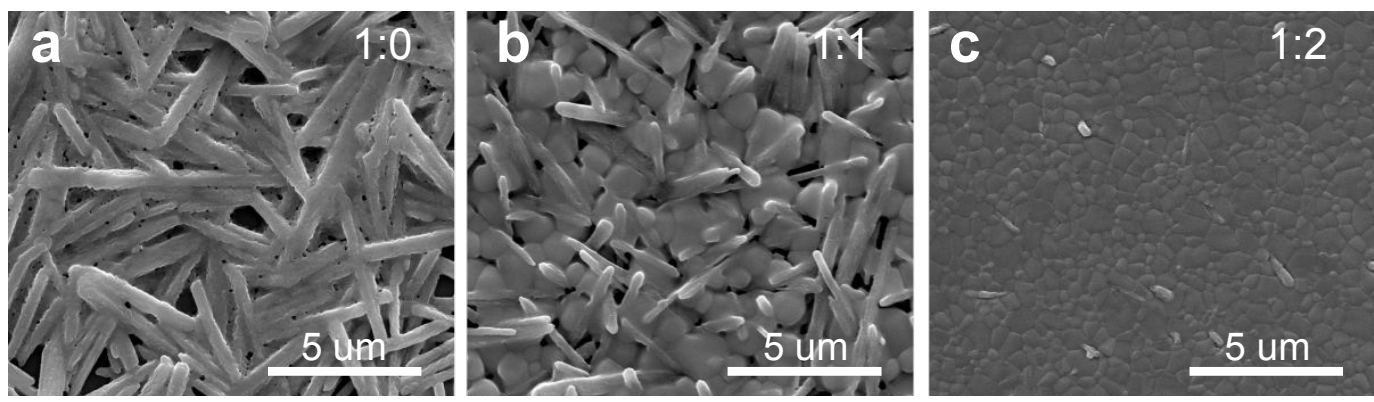

Figure S4. SEM images of perovskite films with a thickness of $\approx 500 \mathrm{~nm}$ processed by DMSO/DMF mixed solvent annealing with different volume ratio. (a) 1:0, v/v, DMSO/DMF (that means pure DMSO, $30 \mathrm{ul}$ ), (b) 1:1, v/v, DMSO/DMF, (c) 1:2, v/v, DMSO/DMF.

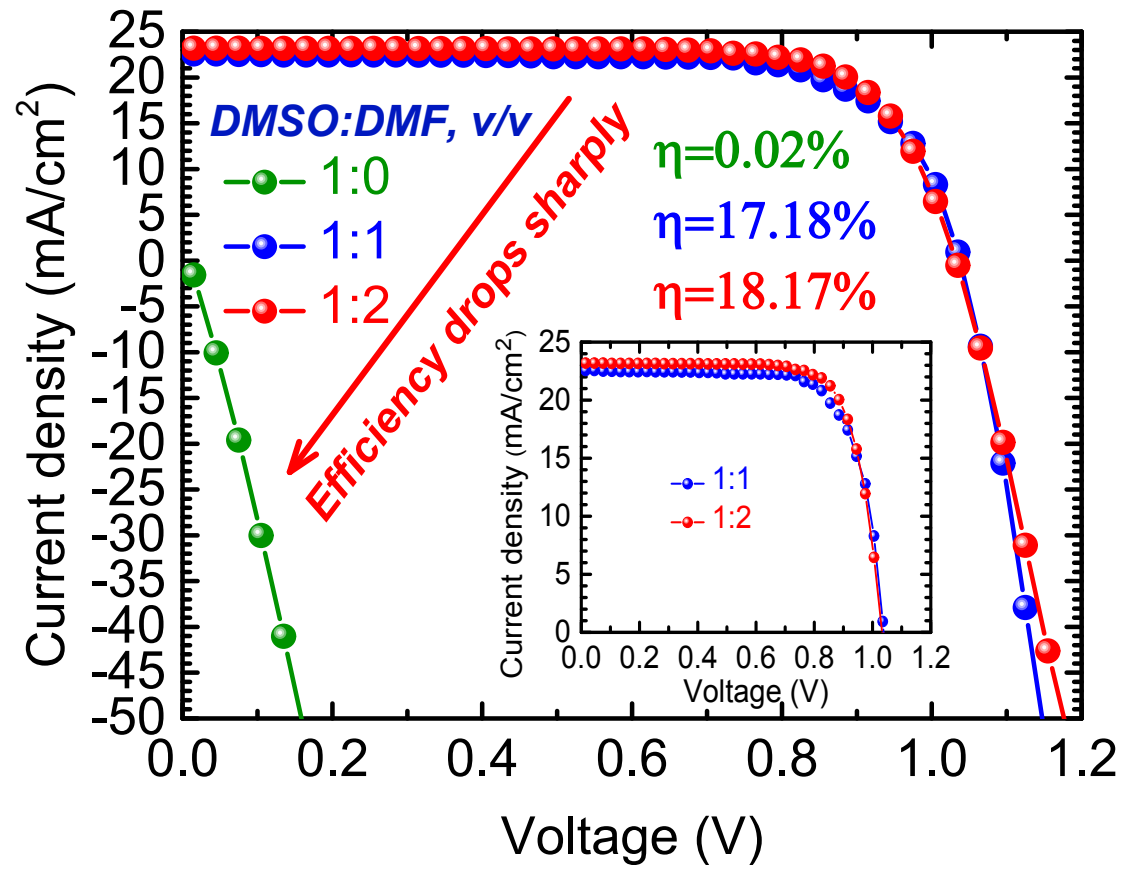

Figure S5. J-V curves of PSCs fabricated based on MSA treated $\mathrm{MAPbI}_{3}$ films with different solvent volume ratio. 

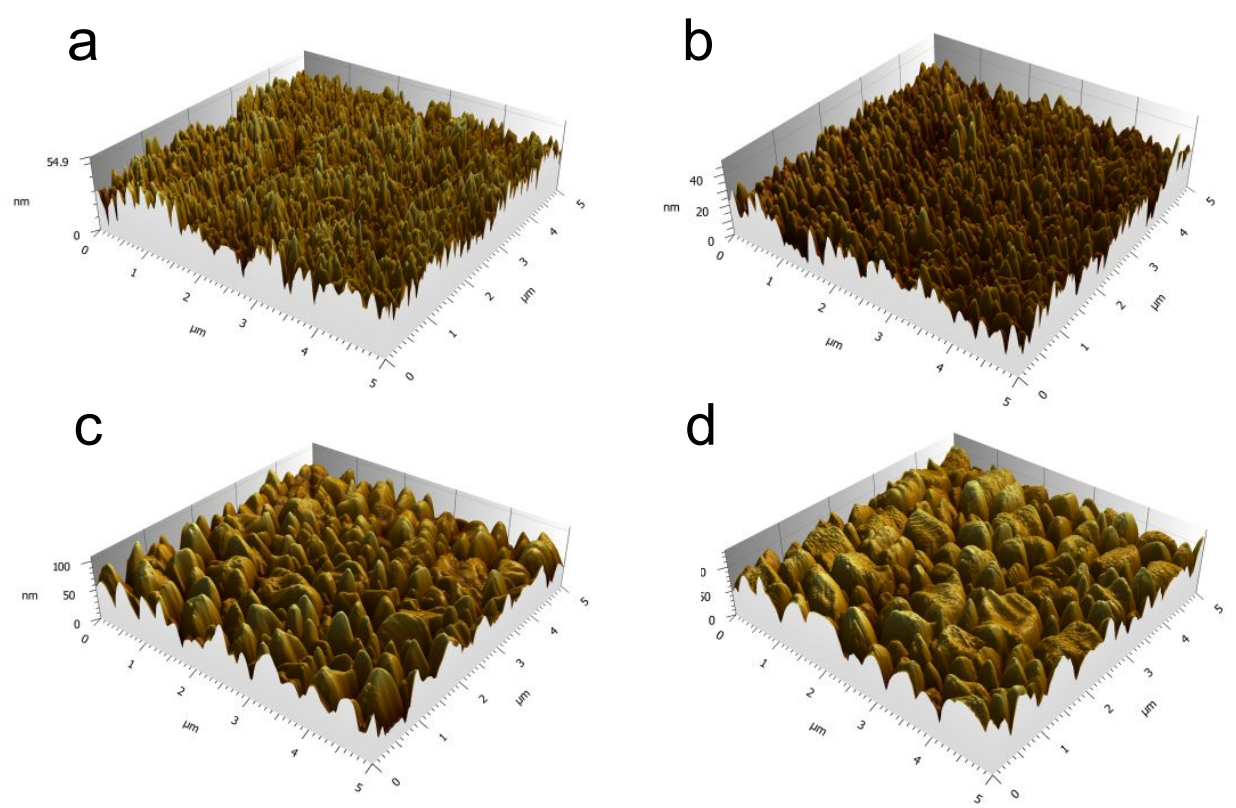

Figure S6. Three dimension AFM images of perovskite films with thickness of $\approx 500$ $\mathrm{nm}$ processed by (a) one-step thermal annealing (OTA), (b) two-step thermal annealing (TTA), (c) pure DMF solvent annealing (PSA) and (d) mixed solvent (DMSO/DMF, 1:9, v/v) annealing (MSA).
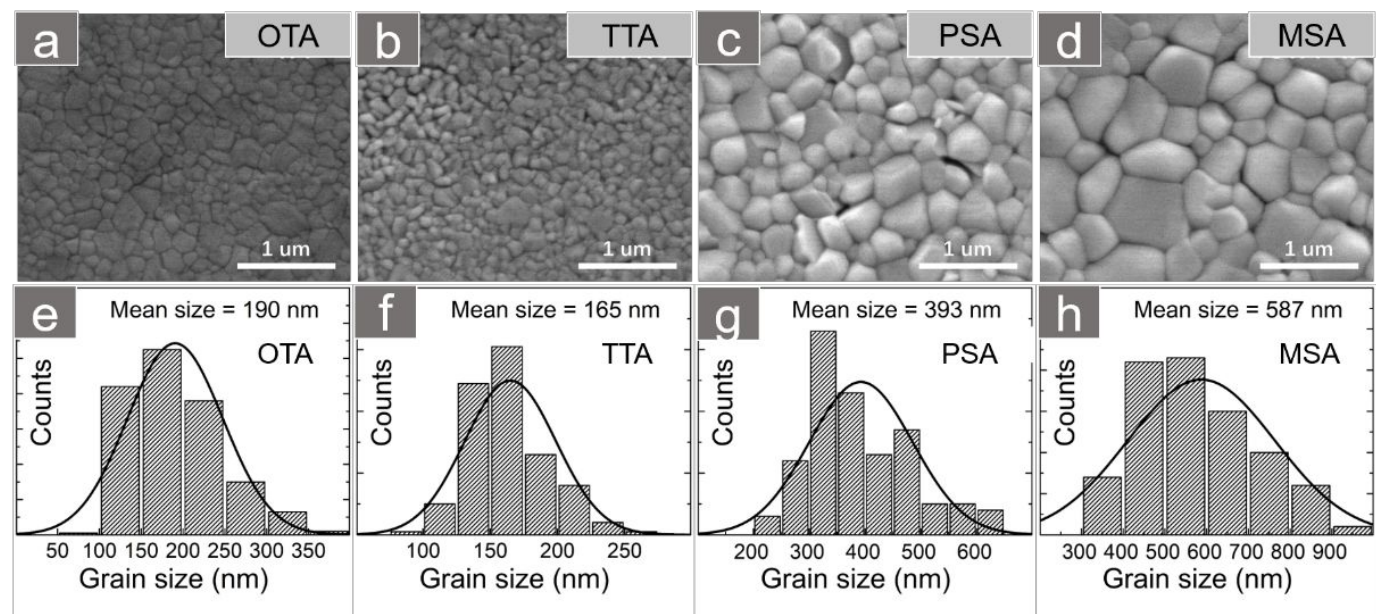

Figure S7. SEM images of perovskite films with a thickness of $\approx 360 \mathrm{~nm}$, and corresponding grain size distribution. (a), (e) OTA, (b), (f) TTA, (c), (g) PSA, (d), (h) MSA (note: the solvent annealing time were lasted for $20 \mathrm{~min}$ ) 


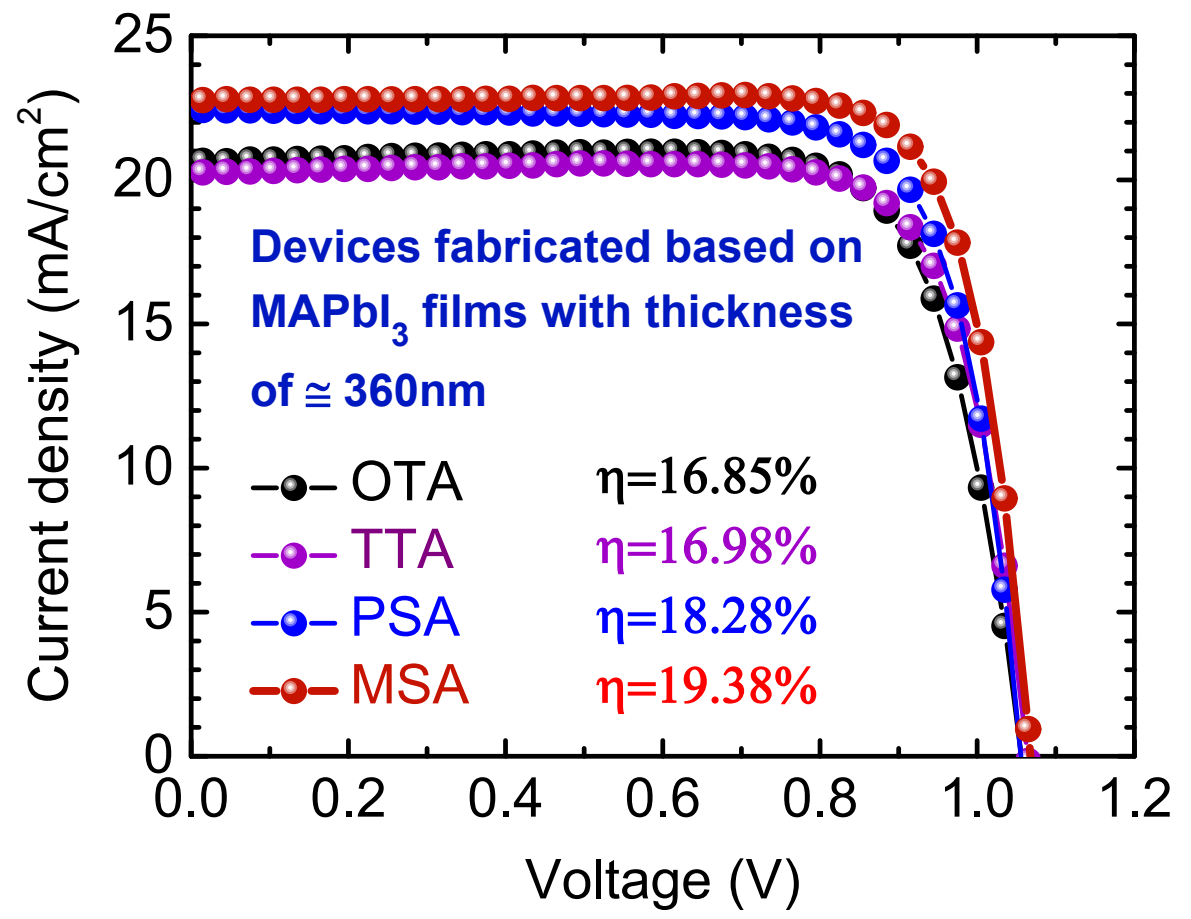

Figure S8. J-V characteristics of solar cells fabricated based on $\mathrm{MAPbI}_{3}$ perovskite films with a thickness of $\approx 360 \mathrm{~nm}$ treated by different annealing strategies (note: the solvent annealing time lasted for $10 \mathrm{~min}$ ), in which OTA devices show performance no better than TTA devices.

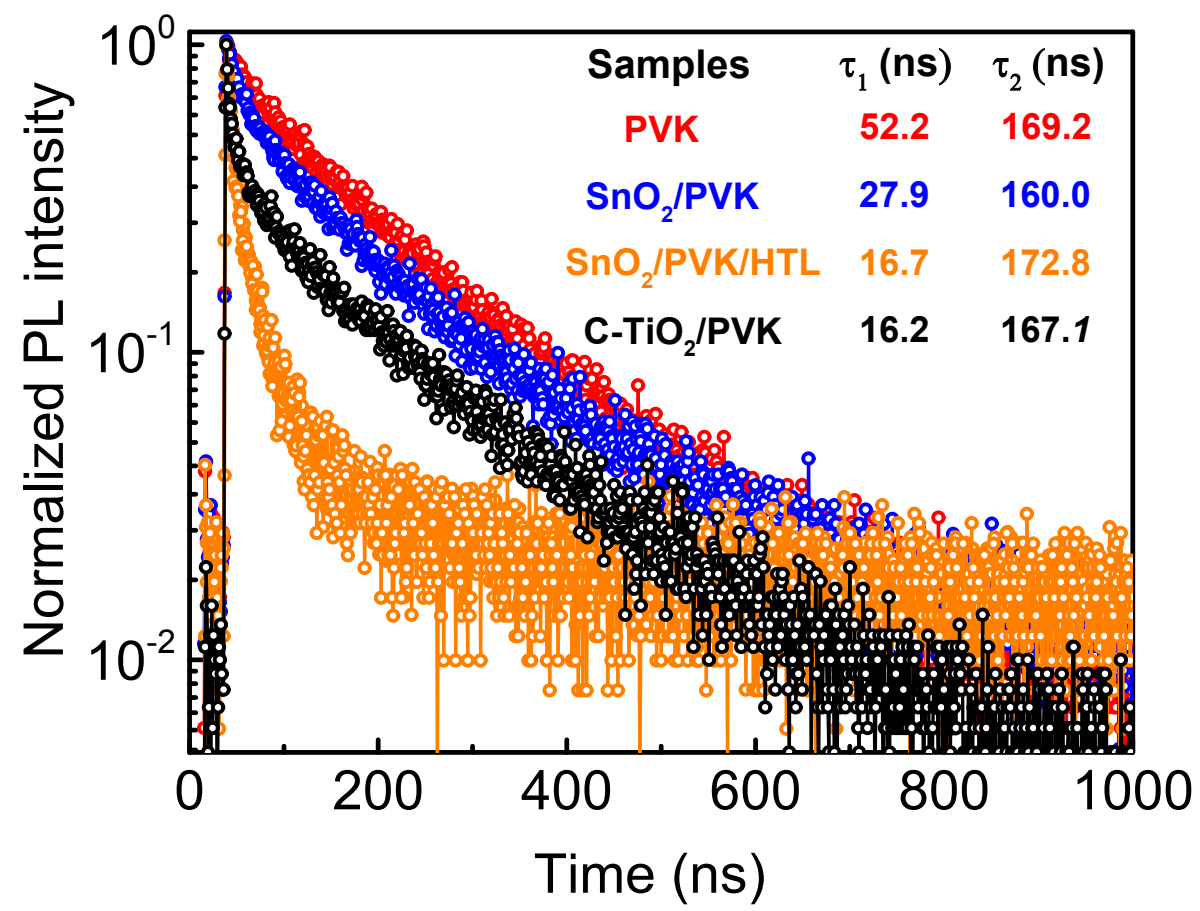

Figure S9. Time-resolved photoluminescence (TRPL) decay spectra of mixed solvent annealing (MSA) treated $\mathrm{MAPbI}_{3}$ perovskite films deposited on different substrates: 
glass/ITO substrate (red, measured through the PVK side), glass/ITO/ $\mathrm{SnO}_{2}$ substrate (blue, measured through the glass side), glass/ITO/ $/ \mathrm{SnO}_{2} / \mathrm{PVK} /$ spiro-OMeTAD (structure, measured through the glass side), glass/FTO/compact $\mathrm{TiO}_{2}$ substrate (black, measured through the glass side).

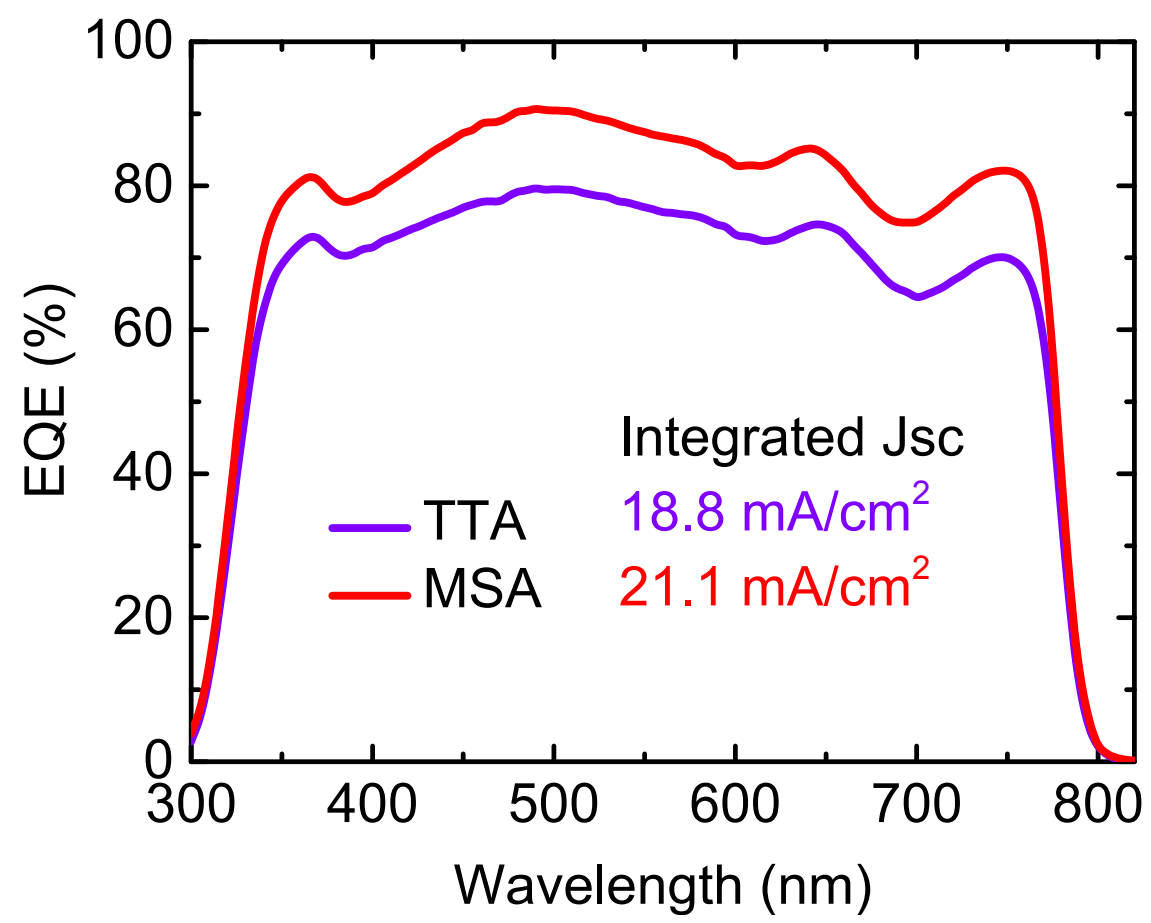

Figure S10. The external quantum efficiency (EQE) spectra of the presentative PSCs fabricated by the TTA and MSA films.

Table S2. Fitted parameter data of Nyquist plots of TTA and MSA devices.

\begin{tabular}{cccccccc}
\hline Device & $\operatorname{Rs}(\Omega)$ & $\operatorname{Rc}(\Omega)$ & $\begin{array}{c}\text { CPE1-T } \\
(\mathrm{F})\end{array}$ & $\begin{array}{c}\text { CPE1-P } \\
(\mathrm{F})\end{array}$ & $\begin{array}{c}\text { Rrec } \\
(\Omega)\end{array}$ & $\begin{array}{c}\text { CPE2-T } \\
(\mathrm{F})\end{array}$ & $\begin{array}{c}\text { CPE2-P } \\
(\mathrm{F})\end{array}$ \\
\hline TTA & 5.00 & 744 & $1.65 \mathrm{E}-9$ & 0.55 & 6500 & $3.91 \mathrm{E}-8$ & 0.94 \\
MSA & 1.95 & 169 & $8.99 \mathrm{E}-10$ & 0.84 & 8375 & $2.75 \mathrm{E}-8$ & 0.97 \\
\hline
\end{tabular}

